\title{
Original
}

\section{The Impact on Quality of Life of Highly Effective Antiemetic Therapy among Breast Cancer Patients Receiving Anthracycline Plus Cyclophosphamide-based Regimen}

\author{
Jumpei ToKumaru ${ }^{1,4)}$, Kazutaka Narui ${ }^{* 2)}$, Akimitsu Yamada ${ }^{3)}$, \\ Kanako KAWAKAMI ${ }^{1)}$, Mariko KAMITE ${ }^{1)}$, Satoko HANDA ${ }^{4)}$, \\ Shinya Hashimoto ${ }^{1)}$, Yasuhisa $\mathrm{KATO}^{4)}$ and Takashi IshikawA ${ }^{5)}$
}

\begin{abstract}
Treatment for chemotherapy-induced nausea and vomiting (CINV) has improved significantly with the development of antiemetic drugs. We conducted a prospective observational study to clarify the quality of life (QOL) impact of antiemetic therapy recommended by the Japanese Cancer Therapy Association (JSCO) guidelines for Japanese breast cancer patients receiving an anthracycline plus cyclophosphamide regimen (ACR). This was an open, single-center, prospective observational study conducted in Yokohama City University Medical Center. Antiemetic therapy recommended by the JSCO guidelines was implemented for all cases treated therein (i.e., aprepitant, dexamethasone, and palonosetron). The primary endpoint was no impact on daily living (NIDL) rate during a 120-hour period following chemotherapy (i.e., overall phase). We use the Japanese version of the Functional Living Index-Emesis (FLIE) to evaluate the impact of CINV on QOL. There were 118 analyzable cases. The NIDL rate during the overall phase was $44.9 \%$, and was significantly lower than the complete response (CR) rate of $58.5 \%$ (i.e., no emetic responses and no rescue medication; $P=0.037$ ). Age $<55$ years $(P=0.008)$ and a history of morning sickness $(P=0.005)$ were identified as independent risk factors of NIDL $(P<0.05)$. Among Japanese breast cancer patients receiving ACR and a combination of aprepitant, dexamethasone, and palonosetron, the NIDL rate was relatively low at approximately $45 \%$. A more effective antiemetic therapy should therefore be developed for patients' QOL that takes NIDL risk factors into account. In addition, our results suggested that the $\mathrm{CR}$ rate is insufficient for evaluating the effect of antiemetic therapy on a patient's QOL.
\end{abstract}

Key words : breast cancer, chemotherapy-induced nausea and vomiting, quality of life, Functional Living Index Emesis

\footnotetext{
1) Pharmaceutical Department, Yokohama City University Medical Center.

2) Department of Breast and Thyroid Surgery, Yokohama City University Medical Center, 4-57 Urafunecho, Minami-ku, Yokohama 232-0024, Japan.

3) Department of Breast Surgery, Chigasaki Municipal Hospital.

4) Department of Drug Information, Division of Drug Information Analytics, Showa University School of Pharmacy.

5) Department of Breast Disease, Tokyo Medical University Hospital.

* To whom corresponding should be addressed.
} 


\section{Introduction}

Chemotherapy-induced nausea and vomiting (CINV) can have major adverse effects on a patient's quality of life (QOL) $)^{1,2)}$. The development of novel classes of antiemetic drugs such as aprepitant, neurokinin 1 (NK1) receptor antagonists, and palonosetron, a second-generation $5 \mathrm{HT}_{3}$ receptor antagonist ${ }^{3,4)}$, has significantly improved the treatment outcomes for $\mathrm{CINV}$, and several guidelines on recommended antiemetic therapy for the prevention of CINV have been published $^{5-7)}$. Guidelines published in 2010 by the Japanese Cancer Therapy Association (JSCO) ${ }^{8)}$ led to standardized antiemetic therapy in Japan, and anticancer drugs have been classified into four groups based on their risk of inducing emesis, with an optimal antiemetic therapy recommended for each of these groups. For instance, the recommended antiemetic therapy for a highly emetogenic chemotherapy is a combination of aprepitant or fos-aprepitant, dexamethasone, and a $5 \mathrm{HT}_{3}$ receptor antagonist.

The anthracycline plus cyclophosphamide regimen (ACR) is used to treat breast cancer patients and is classified as a highly emetogenic chemotherapy. In addition, the risk of CINV can be influenced by various patient-specific risk factors such as female sex, age $<55$ years, no alcohol intake, and having a history of morning sickness or motion sickness ${ }^{10,11}$.

Objective indicators such as complete response ( $\mathrm{CR}$; i.e., no emetic responses and no use of rescue medication) and complete control ( $\mathrm{CC}$; i.e., no emetic responses and no nausea) are widely used in the symptom assessment of CINV. Then in 2009, the US Food and Drug Administration published the "Guidance for industry: patient-reported outcome measures: use in medical product development to support labeling claims" ${ }^{12)}$, a set of guidelines ${ }^{5-7)}$ that recommend the use of patient-reported outcomes (PROs). For outpatient chemotherapy, it is also important to consider the subjective patient-reported and daily life outcomes alongside the objective indexes ${ }^{13)}$, and many studies have employed the Functional Living Index-Emesis (FLIE) ${ }^{14,15)}$ to evaluate the QOL impact of CINV subjectively. Indeed, the Japanese version of the FLIE has shown good reliability and validity ${ }^{16}$, and several survey studies using the FLIE have demonstrated that CINV leads to a notable decrease in $\mathrm{QOL}^{13,17,18)}$. Nevertheless, there has not yet been a detailed report on the QOL impacts of the antiemetic therapy recommended by the JSCO guidelines (a combination of aprepitant, palonosetron, and dexamethasone), with their improved treatment outcomes, for Japanese breast cancer patients receiving a highly emetogenic chemotherapy.

Therefore, herein we conducted a prospective observational study to clarify the QOL impact and reported risk factors of the antiemetic therapy recommended by the JSCO guidelines for Japanese breast cancer patients receiving ACR. We also compared the QOL impact by FLIE, with the $\mathrm{CR}$ or the $\mathrm{CC}$ rates as objective indicators.

\section{Patients and methods}

\section{Study design}

This was an open, single-center, prospective observational study involving a patient diary and conducted at the Yokohama City University Medical Center. The protocol was approved by 
the Research Ethics Committee of Yokohama City University Medical Center, and the study was conducted in line with the Declaration of Helsinki (as revised in October 2013). This trial was registered with the University Hospital Medical Information Network (UMIN) clinical trial registry (UMIN000018544).

\section{Patient selection}

All patients received the combination of antiemetics recommended in the 2010 JSCO Guidelines for Antiemetics in Oncology ${ }^{8)}$. This treatment is targeted for patients undergoing chemotherapy regimens of FEC (fluorouracil $500 \mathrm{mg} / \mathrm{m}^{2}+$ epirubicin $100 \mathrm{mg} / \mathrm{m}^{2}+$ cyclophosphamide $500 \mathrm{mg} / \mathrm{m}^{2}$ ), EC (epirubicin $90 \mathrm{mg} / \mathrm{m}^{2}+$ cyclophosphamide $600 \mathrm{mg} / \mathrm{m}^{2}$ ), and AC (doxorubicin $60 \mathrm{mg} / \mathrm{m}^{2}+$ cyclophosphamide $600 \mathrm{mg} / \mathrm{m}^{2}$ ). Enrolled patients had to fulfill the following inclusion criteria: (a) $\geq 18$ years of age and receiving a highly emetogenic chemotherapy that includes cyclophosphamide and anthracycline for the first time; (b) no experience of nausea or vomiting within 24 hours before receiving an anticancer agent; (c) provided written informed consent; (d) had an Eastern Cooperative Oncology Group Performance Status of 0-2; and (e) capable of understanding all study procedures and completing a patient diary. The exclusion criteria were as follows: (a) presence of brain metastases; (b) presence of hypercalcemia; (c) presence of gastrointestinal obstruction; and (d) taking drugs that can influence the antiemetic effect or participating in other studies involving protocols other than standard antiemetic agents.

\section{Antiemetic treatment}

The $5 \mathrm{HT}_{3}$ receptor antagonist called palonosetron has a superior inhibitory effect on $\mathrm{CINV}$ in the delayed phase compared to another such drug, granisetron ${ }^{9)}$, thus we used palonosetron in this study. During the first course of chemotherapy, all patients received oral aprepitant $125 \mathrm{mg}$, intravenous dexamethasone $9.9 \mathrm{mg}$, and intravenous palonosetron $0.75 \mathrm{mg}$ before chemotherapy on day 1. On days 2 and 3, patients received oral aprepitant $80 \mathrm{mg}$ and oral dexamethasone $8 \mathrm{mg}$. On day 4, patients received oral dexamethasone $8 \mathrm{mg}$. Patients were also prescribed oral prochlorperazine $5 \mathrm{mg}$ as a rescue antiemetic medication, to be used only when nausea and vomiting developed over the 120-hour observation period. We focused solely on the first course of chemotherapy, and patients were allowed to change their antiemetic therapy from the second course onwards.

\section{Outcome assessment}

The acute phase was defined as 0-24 hours following chemotherapy, the delayed phase as 24-120 hours following chemotherapy, and the overall phase as 0-120 hours following chemotherapy. Patients completed a patient diary once per day from days 1 to 5, wherein they recorded emetic episodes, intensity of nausea, and number of times that a rescue antiemetic was needed. Patients also completed the FLIE in order to assess their QOL from days 1 to 5.

Vomiting was defined as one or more emetic episodes or retching (dry heaves), and episodes were considered as separate if they occurred at least $5 \mathrm{~min}$ apart. Nausea intensity was evaluated 
on a 4-point Likert-type scale ( 0 , none; 1 , mild ; 2 , moderate ; 3 , severe) once a day from days 1 to 5 , with significant nausea defined by a rating of 2 or more.

We used the Japanese version of the FLIE to assess the impact of CINV on QOL ${ }^{16)}$. The FLIE is a modified version of the Functional Living Index-Cancer, which is a representative measure of QOL for cancer patients with established reliability and validity ${ }^{14)}$. The FLIE contains 18 items assessing the effect of CINV on patients' daily lives, and contains separate domains for the impacts of nausea and vomiting. The first item in each domain asks the patient to rate how much nausea (vomiting) they have experienced, and then the remaining eight items assess the impact of nausea (vomiting) on the following aspects of a patient's daily life : ability to enjoy meals / liquids, ability to prepare meals / do household tasks, ability to perform daily functions, ability to perform usual recreation, ability to enjoy leisure activities, willingness to spend time with family and friends, extent to which the side effect has caused personal hardship and / or hardship on others.

For each item, participants rate the impact of both nausea and vomiting on daily life, thus producing two scores for each item, and all items are rated on a visual analogue scale (VAS) ranging from 1 ("not at all") to 7 (“a great deal"). The total score ranged from 18 to 126, and we defined a total score of less than 36 as indicating no impact on daily living (NIDL). For each domain, scores ranged from 9 to 63, and we defined a total score of less than 18 as indicating NIDL of nausea or vomiting.

The CR rate was defined as the proportion of patients with no emetic episode and no use of rescue antiemetic medication. Furthermore, the complete control (CC) rate was defined as the proportion of patients with no emetic episode, no use of rescue medication, and no nausea.

The primary endpoint in this study was NIDL rate during the overall study phase. Other endpoints included differences in NIDL, CR, and CC rates in the acute, delayed, and overall

phases. We also investigated the influence of certain risk factors on NIDL rate (including age $<55$ years, no alcohol intake, history of motion sickness, and history of morning sickness) in the acute, delayed, and overall phases.

\section{Statistical analysis}

A $\chi^{2}$ test was used to compare NIDL rate with the $\mathrm{CR}$ or $\mathrm{CC}$ rate in each phase. To assess the influence of the risk factors on NIDL rate, we conducted a multivariate logistic regression analysis with NIDL as the outcome variable and each risk factor as an explanatory variable. We specifically examined whether the risk factors were associated with not achieving NIDL. We also examined the relationship between number of risk factors and NIDL rate using the Cochran-Armitage trend test. Analyses were carried out with SPSS Statistics 24.0, and JMP Pro 12 was used to conduct the Cochran-Armitage trend test. All reported $P$-values were two-sided, and only $P$-values $<0.05$ were considered statistically significant. 


\section{Results}

\section{Patient characteristics}

From June 2014 to December 2016, we enrolled 121 patients were enrolled in the study, and 118 of these were included in the analysis. Three patients were excluded because they either failed to complete the patient diary $(n=2)$ or changed hospitals $(n=1)$. Table 1 lists the characteristics of the analyzed patients. All patients were women, and the median age of patients was 54 years (range 27-76). Across the treatment regimens, 71 patients were on FEC, 44 patients on EC, and 3 patients on AC. The aim of treatments included perioperative adjuvant chemotherapy $(89.0 \%, \mathrm{n}=105)$ and Suppression of advance or recurrence $(11.0 \%, \mathrm{n}=13)$.

Table 1. Patient baseline characteristics

\begin{tabular}{|c|c|c|}
\hline & Number & $\%$ \\
\hline Total & 118 & \\
\hline \multicolumn{3}{|l|}{ Gender } \\
\hline Female & 118 & 100.0 \\
\hline \multicolumn{3}{|l|}{ Aim of chemotherapy } \\
\hline Adjuvant chemotherapy & 25 & 21.2 \\
\hline Neoadjuvant chemotherapy & 80 & 67.8 \\
\hline Suppression of advanced or recurrence & 13 & 11.0 \\
\hline \multicolumn{3}{|l|}{ Age (year) } \\
\hline Median（range） & \multicolumn{2}{|c|}{$54(27-76)$} \\
\hline \multicolumn{3}{|l|}{ Body mass index } \\
\hline Median (range) & \multicolumn{2}{|c|}{$23.4(15.2-44.3)$} \\
\hline \multicolumn{3}{|l|}{ Regimen of chemotherapy } \\
\hline FEC & 71 & 60.2 \\
\hline EC & 44 & 37.3 \\
\hline $\mathrm{AC}$ & 3 & 2.5 \\
\hline \multicolumn{3}{|l|}{ Subtype } \\
\hline $\mathrm{ER}(+)$ & 65 & 55.1 \\
\hline HER2（+） & 40 & 33.9 \\
\hline \multicolumn{3}{|l|}{ Clinical stage } \\
\hline IV & 12 & 10.2 \\
\hline \multicolumn{3}{|l|}{ Risk factor } \\
\hline Age $<55$ & 46 & 39.0 \\
\hline No alcohol intake & 34 & 28.8 \\
\hline History of motion sickness & 22 & 18.6 \\
\hline History of morning sickness & 62 & 52.5 \\
\hline
\end{tabular}

FEC : Fluorouracil + Epirubicin + Cyclophosphamide,

EC : Epirubicin + Cyclophosphamide,

$\mathrm{AC}$ : Doxorubicin + Cyclophosphamide 
Table 2. Number of patients who experienced nausea, vomiting, and use of rescue medication by phase

\begin{tabular}{|c|c|c|c|c|c|c|}
\hline & \multicolumn{2}{|c|}{ acute phase } & \multicolumn{2}{|c|}{ delayed phase } & \multicolumn{2}{|c|}{ overall phase } \\
\hline & $\mathrm{N}$ & $\%$ & $\mathrm{~N}$ & $\%$ & $\mathrm{~N}$ & $\%$ \\
\hline Vomiting & 26 & 22.0 & 17 & 14.4 & 29 & 24.6 \\
\hline Nausea & 83 & 70.3 & 84 & 71.2 & 94 & 79.7 \\
\hline Significant nausea* & 31 & 26.3 & 20 & 16.9 & 36 & 30.5 \\
\hline Use of rescue medication & 33 & 20.0 & 34 & 28.8 & 46 & 39.0 \\
\hline
\end{tabular}

* Significant nausea was defined as nausea with a severity of greater than 2 on the Likert scale.

\section{Antiemetic outcomes}

The antiemetic outcomes are shown in Table 2. The acute phase had the highest rates of vomiting and significant nausea, and they tended to decrease with time.

\section{Impact on $Q O L$}

Figure 1 details the FLIE-based QOL assessment results. During the overall phase, the prevalence of patients with NIDL was $53(44.9 \%)$, and in all phases, the NIDL of nausea tended to be lower than that of vomiting. Figure 2 shows the comparison between the NIDL rate and the $\mathrm{CR}$ or $\mathrm{CC}$ rate. The NIDL rate was significantly lower than the $\mathrm{CR}$ rate in all phases, but was significantly higher than the $\mathrm{CC}$ rate in all phases.

\section{Risk factors}

Table 3 shows the univariate comparisons of NIDL rate by the presence or absence of risk factors for the different phases. The results indicated that age $<55$ years and history of morning sickness were significantly associated with a low NIDL rate in the acute, delayed, and overall phases. The multivariate logistic regression analyses are also detailed in Table 3. Again, age $<55$ years and a history of morning sickness were extracted as independent risk factors of a failure to achieve NIDL $(P<0.05)$.

Table 4 shows the relationship between the number of reported risk factors and NIDL rate in each phase. The NIDL rate tended to decrease significantly as the number of reported risk factors increased in all three phases $(P<0.05)$.

\section{Discussion}

This was a single-center, prospective, observational study on the QOL impact of aprepitant, dexamethasone, and palonosetron combination therapy for ACR in Japanese breast cancer patients. Notably, the NIDL rates in this study remained at $44.9 \%$ in the overall phase (Figure 1).

In Japan, the most recommended antiemetic therapy for a highly emetogenic chemotherapy is the combination of NK-1 receptor antagonist, dexamethasone, and $5 \mathrm{HT}_{3}$ receptor antagonist (preferred one is palonosetron) ${ }^{19)}$. The $\mathrm{CR}$ rate in the overall phase of a randomized doubleblind comparative study on the effectiveness of this particular therapy among 326 breast cancer 


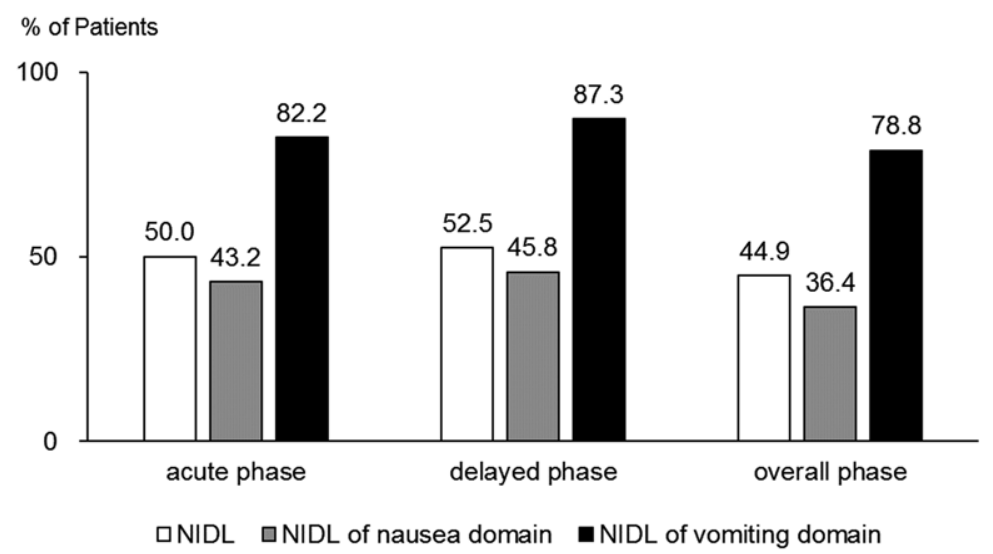

Fig. 1. Percentage of patients with no impact on daily living (NIDL), NIDL in the nausea domain, and NIDL in the vomiting domain

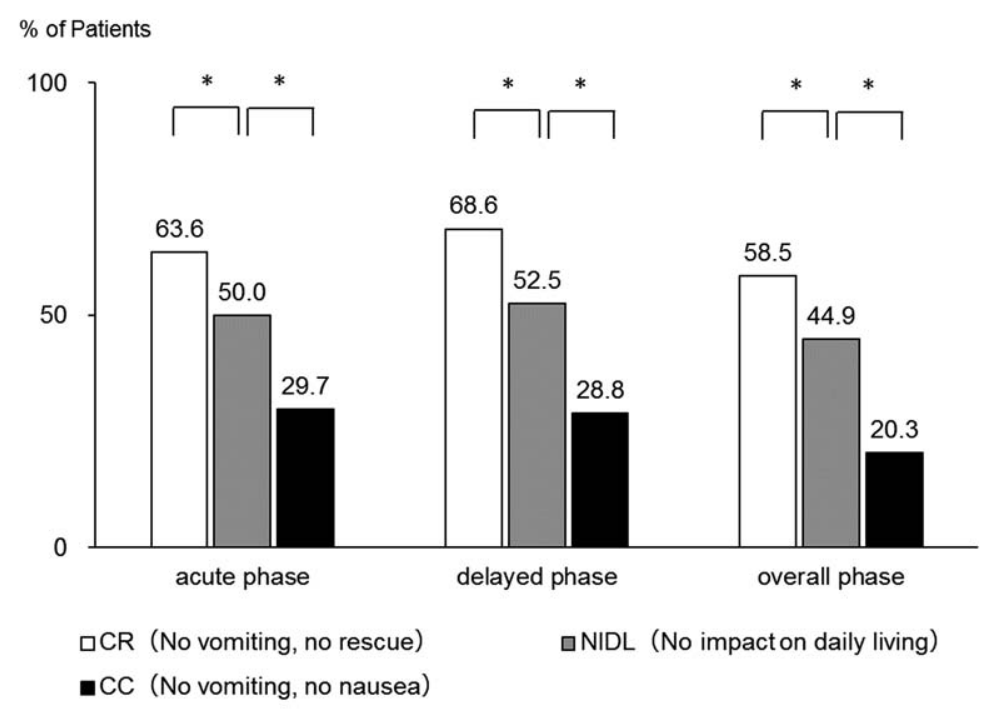

Fig. 2. Percentage of patients with complete response (CR), no impact on daily living (NIDL), and complete control (CC). ${ }^{*} P<0.05$ by $\chi^{2}$ test.

patients undergoing ACR in Japan was $54.9 \%{ }^{20)}$, which is similar to the CR rate of this study. In contrast, another randomized double-blind comparative study ${ }^{9)}$ on the effectiveness of this combination therapy for patients receiving Japanese cisplatin treatment reported a CR rate of $65.7 \%$, which is substantially higher than that of the previous study ${ }^{20)}$ targeting ACR and the present study. In addition, in an integrated analysis of randomized controlled trials ${ }^{21)}$, the incidence of CINV with ACR was higher than that for patients receiving a cisplatin regimen. Therefore, while both cisplatin and ACR are highly emetogenic chemotherapies, the latter seems to need more attention for CINV management when it is used to treat breast cancer.

Several survey studies that investigated QOL using the FLIE questionnaire targeted highly or moderately emetogenic chemotherapy involving a combination of corticosteroid and $5 \mathrm{HT}_{3}$ receptor 
Table 3. Factors associated with no impact on daily living (NIDL) in the (a) acute phase, (b) delayed phase and (c) overall phase

(a) acute phase

\begin{tabular}{|c|c|c|c|c|c|c|c|}
\hline & \multirow{2}{*}{$\mathrm{N}$} & \multicolumn{2}{|c|}{ NIDL } & \multirow{2}{*}{$\begin{array}{c}\begin{array}{c}\text { Univariate } \\
\text { analysis }\end{array} \\
\mathrm{p}\end{array}$} & \multicolumn{3}{|c|}{ Multivariable analysis } \\
\hline & & $\mathrm{N}$ & $\%$ & & OR & $95 \% \mathrm{CI}$ & $\mathrm{p}$ \\
\hline \multicolumn{8}{|c|}{ Age (year) } \\
\hline$<55$ & 61 & 22 & 36.1 & 0.002 & 0.252 & $0.075-0.665$ & 0.005 \\
\hline $55 \leqq$ & 57 & 37 & 64.9 & & & & \\
\hline \multicolumn{8}{|c|}{ No alcohol intake } \\
\hline$(+)$ & 34 & 17 & 50.0 & 1.000 & 1.070 & $0.388-2.955$ & 0.896 \\
\hline$(-)$ & 84 & 42 & 50.0 & & & & \\
\hline \multicolumn{8}{|c|}{ History of motion sickness } \\
\hline$(+)$ & 22 & 8 & 36.4 & 0.156 & 0.691 & $0.210-2.269$ & 0.542 \\
\hline$(-)$ & 96 & 51 & 53.1 & & & & \\
\hline \multicolumn{8}{|c|}{ History of morning sickness } \\
\hline$(+)$ & 62 & 24 & 38.7 & 0.013 & 0.241 & $0.082-0.706$ & 0.009 \\
\hline$(-)$ & 25 & 17 & 68.0 & & & & \\
\hline
\end{tabular}

(b) delayed phase

\begin{tabular}{|c|c|c|c|c|c|c|c|}
\hline & \multirow{2}{*}{$\mathrm{N}$} & \multicolumn{2}{|c|}{ NIDL } & \multirow{2}{*}{$\begin{array}{c}\text { Univariate } \\
\text { analysis } \\
\mathrm{p}\end{array}$} & \multicolumn{3}{|c|}{ Multivariable analysis } \\
\hline & & $\mathrm{N}$ & $\%$ & & OR & $95 \% \mathrm{CI}$ & $\mathrm{p}$ \\
\hline \multicolumn{8}{|c|}{ Age (year) } \\
\hline$<55$ & 61 & 26 & 42.6 & 0.026 & 0.309 & $0.120-0.799$ & 0.015 \\
\hline $55 \leqq$ & 57 & 36 & 63.2 & & & & \\
\hline \multicolumn{8}{|c|}{ No alcohol intake } \\
\hline$(+)$ & 34 & 18 & 52.9 & 0.956 & 1.070 & $0.394-2.908$ & 0.894 \\
\hline$(-)$ & 84 & 44 & 52.4 & & & & \\
\hline \multicolumn{8}{|c|}{ History of motion sickness } \\
\hline$(+)$ & 22 & 10 & 45.5 & 0.460 & 0.954 & $0.301-3.029$ & 0.937 \\
\hline$(-)$ & 96 & 52 & 54.2 & & & & \\
\hline \multicolumn{8}{|c|}{ History of morning sickness } \\
\hline$(+)$ & 62 & 24 & 38.7 & 0.013 & 0.253 & $0.089-0.721$ & 0.010 \\
\hline$(-)$ & 25 & 17 & 68.0 & & & & \\
\hline
\end{tabular}

(c) overall phase

\begin{tabular}{|c|c|c|c|c|c|c|c|}
\hline & \multirow{2}{*}{$\mathrm{N}$} & \multicolumn{2}{|c|}{ NIDL } & \multirow{2}{*}{$\begin{array}{c}\text { Univariate } \\
\text { analysis }\end{array}$} & \multicolumn{3}{|c|}{ Multivariable analysis } \\
\hline & & $\mathrm{N}$ & $\%$ & & OR & $95 \% \mathrm{CI}$ & $\mathrm{p}$ \\
\hline \multicolumn{8}{|c|}{ Age (year) } \\
\hline$<55$ & 61 & 20 & 32.8 & 0.006 & 0.252 & $0.091-0.695$ & 0.008 \\
\hline $55 \leqq$ & 57 & 33 & 57.9 & & & & \\
\hline \multicolumn{8}{|c|}{ No alcohol intake } \\
\hline$(+)$ & 34 & 16 & 47.1 & 0.766 & 1.263 & $0.445-3.583$ & 0.661 \\
\hline$(-)$ & 84 & 37 & 44.0 & & & & \\
\hline \multicolumn{8}{|c|}{ History of motion sickness } \\
\hline$(+)$ & 22 & 6 & 27.3 & 0.065 & 0.436 & $0.118-1.610$ & 0.213 \\
\hline$(-)$ & 96 & 47 & 49.0 & & & & \\
\hline \multicolumn{8}{|c|}{ History of morning sickness } \\
\hline$(+)$ & 62 & 20 & 32.3 & 0.009 & 0.210 & $0.071-0.625$ & 0.005 \\
\hline$(-)$ & 25 & 16 & 64.0 & & & & \\
\hline
\end{tabular}


Table 4. The number of risk factors associated with no impact on daily living (NIDL)

\begin{tabular}{|c|c|c|c|c|c|c|c|}
\hline \multirow{3}{*}{ Number of risk factors } & \multirow{3}{*}{ Number of patients } & \multicolumn{6}{|c|}{ NIDL } \\
\hline & & \multicolumn{2}{|c|}{ acute phase } & \multicolumn{2}{|c|}{ delayed phase } & \multicolumn{2}{|c|}{ overall phase } \\
\hline & & $\mathrm{N}$ & $\%$ & $\mathrm{~N}$ & $\%$ & $\mathrm{~N}$ & $\%$ \\
\hline 0 & 15 & 14 & 93.3 & 13 & 86.7 & 13 & 86.7 \\
\hline 1 & 47 & 26 & 55.3 & 27 & 57.4 & 24 & 51.1 \\
\hline 2 & 24 & 13 & 54.2 & 14 & 58.3 & 11 & 45.8 \\
\hline \multirow[t]{2}{*}{3} & 25 & 5 & 20.0 & 6 & 24.0 & 4 & 16.0 \\
\hline & & \multicolumn{2}{|c|}{${ }^{*} \mathrm{p}<0.05$} & \multicolumn{2}{|c|}{$* \mathrm{p}<0.05$} & \multicolumn{2}{|c|}{${ }^{*} \mathrm{p}<0.05$} \\
\hline
\end{tabular}

${ }^{*}$ Cochran-Armitage tests.

antagonist. Most studies have reported a decrease in QOL by CINV ${ }^{13,17,18)}$. Among these, Fernández-Ortega et $a l^{13)}$ analyzed 160 patients treated with highly or moderately emetogenic chemotherapy with steroids and $5 \mathrm{HT}_{3}$ receptor antagonist as CINV prophylaxis, and reported that QOL was not affected by nausea in $55 \%$ of patients. In another study, Ballatori et al ${ }^{18)}$ evaluated 152 patients receiving cisplatin followed by appropriate prophylaxis according to Multinational Association of Supportive Care in Cancer (MASCC) guidelines and reported QOL effects due to vomiting in $67.3 \%$ of the patients and due to nausea in $76.6 \%$ of the patients. For the first time in this study, we targeted Japanese patients with breast cancer receiving an ACR regimen of aprepitant, dexamethasone, and palonosetron, and investigated QOL using FLIE questionnaires. And we found that NIDL for nausea was $36.4 \%$ and NIDL for vomiting was $78.8 \%$ in the overall phase. Therefore, we found no significant improvement in QOL compared to previous studies.

We also found that the incidence of significant nausea during the overall phase was higher than the incidence of vomiting $(30.5 \%$ vs. $24.6 \%)$, and that NIDL could not be achieved for nausea $(36.4 \%)$, but could generally be achieved for vomiting $(78.8 \%)$. Together, these results indicate that the influence of nausea on QOL was considerable. Furthermore, the NIDL rate, which is an index of QOL, was significantly lower than the CR rate, possibly because nausea was not included as an evaluation indicator when measuring $\mathrm{CR}$ rate. A previous study investigating the effect of CINV on QOL similarly presumed that nausea has a greater negative impact on QOL than does vomiting because vomiting is a short-term event, whereas nausea is a prolonged feeling ${ }^{13)}$; however, since the NIDL rate in this study was significantly higher than the $\mathrm{CC}$ rate (no emetic responses and no nausea), we might infer that the $\mathrm{CC}$ rate is not the most ideal goal of antiemetic therapy and that mild nausea probably did not lead to a lower QOL.

By contrast, significant nausea might have a large influence on QOL, and our results indeed suggest that the CR rate is insufficient for evaluating the QOL effects of antiemetic therapy. Consequently and with the growing use of outpatient chemotherapy, proper assessment of QOL using subjective indexes, including nausea, is essential for CINV management, and it might be necessary to calculate NIDL rates using the FLIE to effectively evaluate the impact of CINV on patient QOL. 
One previous study investigating CINV and QOL among breast cancer patients undergoing ACR conducted in Canada, which also used the FLIE, reported a CR rate of $51 \%$ and an NIDL rate of $63.5 \%{ }^{22)}$; also, in contradiction to the present study results, the NIDL rate was higher than the CR rate. A previous study in Japan using the FLIE Japanese version attributed this difference in rates to the period used to define NIDL ${ }^{23)}$; in the Canadian study, the FLIE was obtained 120 hours following treatment, and participants were asked to rate the last 5 days, whereas in the FLIE Japanese version, the questionnaire was administered to patients every 24 hours. In addition, the FLIE is a subjective evaluation of QOL and thus possibly affected by memory, which is likely to become increasingly ambiguous over time. Thus, the FLIE Japanese version used in our study might more accurately measure $\mathrm{QOL}^{23)}$.

For Japanese patients receiving ACR for breast cancer, the NIDL rate remained at about $50 \%$, despite the use of effective antiemetics. This might be because a large number of the cases had risk factors of CINV, such as being young and female, and in this study, the NIDL rate of individuals aged $<55$ years or with a history of morning sickness was significantly lower (Table 3). In addition, the NIDL rate tended to decrease as the number of known risk factors increased in this study (Table 4). On the other hand, the NIDL rate was over $90 \%$ for cases without any risk factors. Thus, the antiemetic therapy seemed adequate only when no risk factors were present. Therefore, individualized antiemetic therapy taking risk factors into account should be considered. Recently, the usefulness ${ }^{24)}$ of combined antiemetic treatment of olanzapine with aprepitant, dexamethasone, and a $5 \mathrm{HT}_{3}$ receptor antagonist has been demonstrated. Thus, patients with many risk factors might improve their QOL by adding olanzapine in conjunction with other antiemetics.

Since this study is a single-arm, single-center observational study, we cannot deny the possibility of omitting other confounders affecting QOL, or the possibility of influencing factors within the treatment environment. Thus, a more detailed and larger-scale study is warranted.

In summary, among Japanese patients with breast cancer receiving ACR, even usiug the recommended combination therapy of aprepitant, dexamethasone, and palonosetron, the NIDL rate was only about $45 \%$. It is therefore necessary to develop a more effective antiemetic therapy that takes patient-level risk factors into account to improve overall patient QOL. In addition, the NIDL rate was significantly lower than the CR rate in this study, suggesting that the CR rate is insufficient for evaluating the effect of antiemetic therapy on a patient's QOL.

\section{Acknowledgments}

This work was supported by Grants-in-Aid for Scientific Research from the Japan Society for the Promotion of Science (JSPS) (Grant Number 16K19055 for AY).

\section{Compliance with Ethical Standards}

Conflicts of interest

No potential conflicts of interest to disclose. 
Ethical approval

All procedures performed in studies involving human participants were in accordance with the ethical standards of the institutional and/or national research committee and with the 1964 Helsinki declaration and its later amendments or comparable ethical standards.

Informed consent

Informed consent was obtained from all individual participants included in the study.

\section{References}

1) de Boer-Dennert M, de Wit R, Schmitz PI, et al. Patient perceptions of the side-effects of chemotherapy: the influence of 5HT3 antagonists. Br J Cancer. 1999;76:1055-1061.

2) Coates A, Abraham S, Kaye SB, et al. On the receiving end-patient perception of the side-effects of cancer chemotherapy. Eur J Cancer Clin Oncol. 1983;19:203-208.

3) Hesketh PJ, Grunberg SM, Gralla RJ, et al. The oral neurokinin-1 antagonist aprepitant for the prevention of chemotherapy-induced nausea and vomiting: a multinational, randomized, double-blind, placebo-controlled trial in patients receiving high-dose cisplatin-the Aprepitant Protocol 052 Study Group. J Clin Oncol. 2003;21:4112-4119.

4) Saito M, Aogi K, Sekine I, et al. Palonosetron plus dexamethasone versus granisetron plus dexamethasone for prevention of nausea and vomiting during chemotherapy: a double-blind, double-dummy, randomised, comparative phase III trial. Lancet Oncol. 2009;10:115-124.

5) Basch E, Prestrud AA, Hesketh PJ, et al. Antiemetics: American Society of Clinical Oncology clinical practice guideline update. J Clin Oncol. 2011;29:4189-4198. Erratum in: J Clin Oncol. 2014;32:2117.

6) Roila F, Herrstedt J, Aapro M, et al. Guideline update for MASCC and ESMO in the prevention of chemotherapy- and radiotherapy-induced nausea and vomiting: results of the Perugia consensus conference. Ann Oncol. 2010;21(suppl 5):v232-v243.

7) National Comprehensive Cancer Network. NCCN clinical practice guidelines in oncology: antiemesis. V.1.2015 (Internet). (accessed 2015 Apr 14) Available from: http://www.nccn.org/professionals/phsician_gis/pdf/antiemesis.pdf

8) Japan Society of Clinical Oncology. JSCO clinical practice guidelines for antiemetics Tokyo: Kanehara Shuppan; 2010. (in Japanese).

9) Suzuki K, Yamanaka T, Hashimoto H, et al. Randomized, double-blind, phase III trial of palonosetron versus granisetron in the triplet regimen for preventing chemotherapy-induced nausea and vomiting after highly emetogenic chemotherapy: TRIPLE study. Ann Oncol. 2016;27:1601-1606.

10) Hesketh PJ, Aapro M, Street JC, et al. Evaluation of risk factors predictive of nausea and vomiting with current standard-of-care antiemetic treatment: analysis of two phase III trial fosaprepitant in patients receiving cisplatinbased chemotherapy. Support Care Cancer. 2010;18:1171-1177.

11) Warr DG, Street JC, Carides AD, et al. Evaluation of risk factors predictive of nausea and vomiting with current standard-of-care antiemetic treatment: analysis of phase 3 trial of aprepitant in patients receiving adriamycincyclophosphamide-based chemotherapy. Support Care Cancer. 2011;19:807-813.

12) U.S. Department of Health and Human Services Food and Drug Administration, Center for Drug Evaluation and Research (CDER), Center for Biologics Evaluation and Research (CBER), et al. Guidance for industry: patientreported outcome measures: use in medical product development to support labeling claims. Rockville: Food and Drug Administration; 2009.

13) Fernandez-Ortega P, Caloto MT, Chirveches E, et al. Chemotherapy-induced nausea and vomiting in clinical practice: impact on patients' quality of life. Support Care Cancer. 2012;20:3141-3148.

14) Lindley CM, Hirsch JD, O’Neill CV, et al. Quality of life consequences of chemotherapy-induced emesis. Qual Life Res. 1992;1:331-340. 
15) Sommariva S, Pongiglione B, Tarricone R. Impact of chemotherapy-induced nausea and vomiting on health-related quality of life and resource utilization: a systematic review. Crit Rev Oncol Hematol. 2016;99:13-36.

16) Satou A, Yamazaki T, Nukariya N, et al. Development of a Japanese version of the FLIE. Jpn J Cancer Chemother. 2002;29:281-291.

17) Bloechl-Daum B, Deuson RR, Mavros P, et al. Delayed nausea and vomiting continue to reduce patients' quality of life after highly and moderately emetogenic chemotherapy despite antiemetic treatment. J Clin Oncol. 2006;24:4472-4478.

18) Ballatori E, Roila F, Ruggeri B, et al. The impact of chemotherapy-induced nausea and vomiting on health-related quality of life. Support Care Cancer. 2007;15:179-185.

19) Japan Society of Clinical Oncology. JSCO clinical practice guidelines for antiemetics. 2nd ed. Tokyo: Kanehara Shuppan; 2015. (in Japanese).

20) Matsumoto K, Takahashi M, Sato K, et al. Palonosetron or granisetron for prevention of CINV in patients with breast cancer receiving dexamethasone and fosaprepitant following anthracycline plus cyclophosphamide (AC) regimen. J Clin Oncol. 2015;33 suppl:abstr 9598.

21) Kubota K, Saito M, Aogi K, et al. Control of nausea with palonosetron versus granisetron, both combined with dexamethasone, in patients receiving cisplatin- or anthracycline plus cyclophosphamide-based regimens. Support Care Cancer. 2016;24:4025-4033.

22) Warr DG, Hesketh PJ, Gralla RJ, et al. Efficacy and tolerability of aprepitant for the prevention of chemotherapyinduced nausea and vomiting in patients with breast cancer after moderately emetogenic chemotherapy. $J$ Clin Oncol. 2005;23:2822-2830.

23) Kawano C, Hirayama T, Koizumi W, et al. A study of objective assessment and quality of life assessment on chemotherapy-induced nausea and vomiting. Jpn J Pharm Palliat Care Sci. 2014;7:89-95. (in Japanese).

24) Navari RM, Qin R, Ruddy KJ, et al. Olanzapine for the prevention of chemotherapy-induced nausea and vomiting. N Engl J Med. 2016;375:134-142.

[Received January 11, 2018 : Accepted January 24, 2018] 